\title{
The Problem of Integral Geometry of Volterra Type with a Weight Function of a Special Type
}

\author{
Akram.H. Begmatov 1,2,", M.E. Muminov ${ }^{3}$, Z.H. Ochilov ${ }^{2}$ \\ ${ }^{1}$ Gwangyang Jecheol High School, South of Korea \\ ${ }^{2}$ Samarkand State University, Uzbekistan \\ ${ }^{3}$ Faculty of Science, Malaysia Technology University, Malaysia
}

Copyright (C) 2015 by authors, all rights reserved. Authors agree that this article remains permanently open access under the terms of the Creative Commons Attribution License 4.0 International License

\begin{abstract}
We study new problem of reconstruction of a function in a strip from their given integrals with known weight function along polygonal lines. We obtained two simply inversion formulas for the solution to the problem. We prove uniqueness and existence theorems for solutions and obtain stability estimates of a solution to the problem in Sobolev's spaces and thus show their weak ill-posedness. Then we consider integral geometry problems with perturbation. The uniqueness theorems are proved and stability estimates of solutions in Sobolev spaces are obtained.
\end{abstract}

Keywords Ill-posed Problems, Integral Geometry Problems, Integral Transforms, Inversion Formula, Uniqueness, Existence Theorem, Weak Instability, Perturbation

\section{Introduction}

The notion of correctness (correct) statement of the problem of mathematical physics emerged in the early twentieth century in the writings of eminent French mathematician Jacques Hadamar [1]. Problems of mathematical physic so boundary problem for equations with partial derivatives called correctly if the following conditions:

1. The solution of the problem exists;

2. The problem is unique;

3. The solution depends continuously on the data of the problem.

When studying the mathematical models of technical tasks naturally reformulate specified conditions in the following form:

A. Solution task exists for all data belonging to some closed space in nor med linear spaces of type $C_{k}, L_{p}, I_{p}, W_{p}^{l}$ and belongs to the same type;

B. Solution the task is only in any analogous space;
C. Infinitely small variations of these tasks in the data space, correspond to infinitely small in the solution space, variations of the solution

The classic example the Tasks, in correctly to Hadamard, is the Cauchy problem for the Laplace equation.

Operator equation of the first kind is called the equation

$$
A x=f
$$

where $x, f$-elements of the spaces $X, A, F$ - compact operator from $X$ to $F$.

The operator $A^{-1}$ is not continuous-the problem of solving equation (1) is incorrect.

The right side of equation (1) is often obtained on the basis of evidence of physical devices. Norm in the space corresponds to the fact, that is known to estimate the maximum measurement error. The norm in $L 1$ represents the mean square error.

The integral geometry problem

$$
\int_{S(y)} g(x, y) f(y) u(x) d s=f(y)
$$

There is the problem of solving the operator equation for the function $u(x)$ under the assumption that we are given the right side $f(y)$, weight function $g(x, y)$ and the variety in which integration is carried out.

The main objective of computed tomographyis an integral geometry problem.

Let $u(x)$ - the coefficient of absorption; $I$ - radiation intensity. Then

$$
\Delta I / I=u(x) \Delta x
$$

Accordingly, the initial intensity of beam $L$, and its intensity after passing through the body.

Integrating this expression, we obtain

$$
\int_{L} u(\xi) d s=f(x), f(x)=\ln I_{0} / I_{1}
$$

Similarly, you can submit a mathematical model of 
seismic tomography and ultrasound imaging.

We denote $v=v(x, y)$ - the speed of signal propagation in the medium, $n=\frac{1}{v}$ - refractive index; $\tau(x, y)-$ the travel time of the signal between points $x$ and $y$ on the surface, the signal path $r=m(x, y)$. Then

$$
\tau=\int_{\Gamma} n(x, y) d s .
$$

The problem of solving equation (2) called weakly ill-posed, if for this problem and its solution of the equation, you can to pick up a such pair Function spaces in the definition of the norm involving a finite number of derivatives that the operator handling for this pair of spaces is continuous[2].

If a pair of spaces does not exist, then the problem is greatly flawed. Of course, this classification is the case not only for the integral geometry problems, but also in the general theory of ill-posed problems.

V.G. Romanov in[5] investigated the questions of uniqueness and stability of solution of integral geometry in the case where the manifold on which integration is carried out, have the kind of paraboloids, weighting functions and diversity are invariant under the group of all the movements along a fixed hyperplane.

Weakly ill-posed integral geometry problems Voltaire type with weight functions which are particularly studied in [6-9], weakly ill-posed integral geometry problems on special curves and surfaces with singularities are studied in [10-13].

The integral geometry problems on the paraboloids with perturbation in three-dimensional layer considered in [13].

\section{Recovering a Function from Polygonal Lines}

Let $\mathrm{G}$-set of bounded functions $g(\cdot)$, defined on $R^{1}$ and satisfy the following conditions:

I. Function I( $\cdot, \cdot)$ definedon $R \times C$ by the formula

$$
\mathrm{I}(\lambda, p)=\int_{0}^{\infty}\left[e^{i \lambda h} g(h)-e^{-i \lambda h} g(-h)\right] e^{-p h} d h,
$$

II. Exist numbers $n, m \in\{0,1,2, \ldots$.$\} and are such that$

$$
\left|\frac{1}{\mathrm{I}(\lambda, p)}\right| \leq C\left(|p|^{n}+|\lambda|^{m}\right)
$$

for all $\lambda \in R$ and $\operatorname{Re} p>\rho$;

Remark. Note that the set $\mathrm{G}$ is notempty. For example, if the function $g(\cdot)$ has the form

$$
g(h)=\left\{\begin{array}{lll}
a_{1} e^{-k h}, & \text { when } & h \geq 0, \\
-a_{1} e^{k h}, & \text { when } & h<0,
\end{array}\right.
$$

$a_{1} k>0$, then

$$
\begin{gathered}
\mathrm{I}(\lambda, p)=2 a_{1} \int_{0}^{\infty} e^{-(p+k) h} \cos (\lambda h) d h= \\
\quad=2 \frac{a_{1}(k+p)}{(k+p)^{2}+\lambda^{2}} \neq 0
\end{gathered}
$$

for all $\lambda \in R$ and $\operatorname{Re} p \geq 0$.

Because

$$
\left|\frac{k+p}{(k+p)^{2}+\lambda^{2}}\right|=\left|\frac{1}{k+p+\frac{\lambda^{2}}{k+p}}\right| \geq \frac{1}{|k+p|+\frac{\lambda^{2}}{|k+p|}},
$$

Where

$$
|k+p|=\sqrt{(k+\operatorname{Re} p)^{2}+(\operatorname{Re} p)^{2}} \geq|k+\operatorname{Re} p|=k+\operatorname{Re} p \geq 1 .
$$

When $k \geq 1 \operatorname{Re} p \geq 0$;

Hence, we obtain

$$
\begin{aligned}
& \left|\frac{k+p}{(k+p)^{2}+\lambda^{2}}\right|=\left|\frac{1}{k+p+\frac{\lambda^{2}}{k+p}}\right| \geq \\
& \geq \frac{1}{|k+p|+\frac{\lambda^{2}}{|k+p|}} \geq \frac{1}{|k|+|p|+\lambda^{2}} \geq \frac{1}{|p|+\lambda^{2}},
\end{aligned}
$$

So

$$
|\mathrm{I}(\lambda, p)| \geq \frac{1}{|p|+\lambda^{2}}
$$

Therefore

$$
\frac{1}{|\mathrm{I}(\lambda, p)|} \geq\left(|p|+\lambda^{2}\right) N
$$

i.e. $\mathrm{C}=\mathrm{N}, \mathrm{n}=1, \mathrm{~m}=2, \mathrm{p}=0, \mathrm{~g} \in \mathrm{G}$.

We introduce the notation:

$$
\begin{array}{ll}
\Omega=\left\{(x, y): x \in R^{1},\right. & y \in(0, l), \quad l<\infty\} \\
\bar{\Omega}=\left\{(x, y): x \in R^{1},\right. & y \in[0, l],\}
\end{array}
$$

Inband $\bar{\Omega}$ is considered a family of scrap $P(x, y)$ that is uniquely parameterized by the coordinates of its vertices

$$
\begin{gathered}
(x, y) \in \bar{\Omega}: P(x, y)=\{(\xi, \eta):(x-y)+(\eta-\xi)=0, \\
0 \leq \eta \leq, x-y \leq \xi \leq x\} \bigcup \\
\{(\xi, \eta):(x+y)-(\eta+\xi)=0,0 \leq \eta \leq y, x \leq \xi \leq x+y\} .
\end{gathered}
$$

Problem I. Required to determine the function of two variables $u(x, y)$, if all of $(x, y)$ the bands $\Omega$ known integrals of functions $u(x, y)$ of Polygonal Lines $P(x, y)$ : 


$$
\begin{gathered}
\int_{x-y}^{x} g(x-\xi) u(\xi, \xi+y-x) d \xi+ \\
+\int_{x}^{x+y} g(x-\xi) u(\xi x+y-\xi) d \xi=f(x, y),
\end{gathered}
$$

where $\mathrm{g}$-weight function of the set G.

Function $u(x, y)$ - is function of class $\mathrm{U}$, which has all continuous partial derivative sup to $(n+m+4)$ - the second order inclusive and finite with the carrier and $R_{+}^{2}$ :

$$
\sup p u \subset D=\{(x, y):-a<x<a, 0<y<l\}
$$

where $\rho<a<\infty, l<\infty$.

$$
\begin{gathered}
I_{1}(\lambda, y-\eta)=\frac{1}{2 \pi i} \int_{a-i \infty}^{a+i \infty} e^{p(y-\eta)} \frac{d p}{p^{n+2} I(\lambda, p)} \\
I_{2}(x-\xi, y-\eta)=\int_{-\infty}^{+\infty} e^{-i \lambda(x-\xi)} \frac{I_{1}(\lambda, h)}{1+\lambda^{m+2}} d \lambda
\end{gathered}
$$

The following theorem holds:

Theorem 1. Suppose that the function $f(x, y)$ is known to all $(x, y)$ of the bands $\Omega$. Then the solution oflin the class $U$ is unique and has the representation

$$
\begin{array}{r}
u(x, y)=\int_{0}^{\infty} \int_{-\infty}^{\infty} I_{2}(x-\xi, y-\eta)\left[\frac{\partial^{n+2}}{\partial \eta^{n+2}}-\right. \\
\left.-\frac{\partial^{n=m+4}}{\partial \xi^{m+2} \partial \eta^{n+2}}\right] f(\xi, \eta) d \xi d \eta
\end{array}
$$

and executed inequality

$$
\|u\|_{L_{2}(\Omega)} \leq C_{0}\|f\|_{W_{2}^{n+2, m+2}(\Omega)},
$$

where $C_{0}$ - some constant.

Proof. For the first integral of equation (7) we introduce the following change of variables:

$$
\xi=x-y+\eta, d \xi=d \eta,
$$

and for the second term of the left side of (7), we apply the following change:

$$
\xi=x+y-\eta, d \xi=-d \eta .
$$

As a result, going to (7) to integration over $d \eta$, obtain the integral

$$
\begin{gathered}
\int_{0}^{y} g(y-\eta) u(x-y+\eta, \eta) d \eta- \\
-\int_{0}^{y} g(\eta-y) u(x+y-\eta, \eta) d \eta=f(x, y)
\end{gathered}
$$

or

$$
\begin{gathered}
\int_{0}^{y} g(h) u(x-h, y-h) d h- \\
-\int_{0}^{y} g(-h) u(x+h, y-h) d h=f(x, y)
\end{gathered}
$$

where $h=y-\eta$.

Applying the Fourier transform to the variable $\mathrm{x}$ to both sides of equation (11) we obtain:

$$
\begin{gathered}
\hat{f}(\lambda, y)=\int_{-\infty}^{+\infty} e^{i \lambda x} * \\
*\left\{\int_{0}^{y} g(h) u(x-h, y-h) d h-\int_{0}^{y} g(-h) u(x+h, y-h) d h\right\} d x= \\
=\int_{-\infty}^{+\infty} e^{i \lambda x \int_{0}^{u} g(h) u(x-h, y-h) d h d x-} \\
-\int_{-\infty}^{+\infty} e^{i \lambda x} \int_{0}^{y} g(-h) u(x+h, y-h) d h d x= \\
=\int_{0}^{y} e^{i \lambda h} g(h)\left\{\int_{-\infty}^{+\infty} e^{i \lambda(x-h)} u(x-h, y-h) d x\right\} d h- \\
-\int_{0}^{y} e^{-i \lambda h} g(-h)\left\{\int_{-\infty}^{+\infty} e^{i \lambda(x+y)} u(x+h, y-h) d x\right\} d h=
\end{gathered}
$$$$
=\int_{0}^{y} e^{i \lambda h} g(h) \hat{u}(\lambda, y-h) d h-\int_{0}^{y} e^{-i \lambda h} g(-h) \hat{u}(\lambda, y-h) d h=
$$$$
=\int_{0}^{y}\left[e^{i \lambda h} g(h)-e^{-i \lambda h} g(-h)\right]_{u}^{\wedge}(\lambda, y-h) d h
$$

That means

$$
\hat{f}(\lambda, y)=\int_{0}^{y}\left[e^{i \lambda h} g(h)-e^{-i \lambda h} g(-h)\right] \hat{u}(\lambda, y-h) d h .
$$

For the function $f(\lambda ;)$ fair enough following:

$1^{0}$. Function $f(\lambda ;)$ satisfies a Holder condition everywhere on R, i.e. 


$$
|\hat{f}(\lambda, y+t)-\hat{f}(\lambda, y)|=\mid \int_{0}^{y+t}\left[e^{i \lambda h} g(h)-e^{-i \lambda h} g(-h)\right] * \quad \hat{\sim} \quad \hat{u}(\lambda, p) I(\lambda, p)=\hat{f}(\lambda, p) .
$$$$
* \hat{u}(\lambda, y-h) d h-\int_{o}^{y}\left[e^{i \lambda h} g(h)-e^{-i \lambda h} g(h)\right] \hat{u}(\lambda, y-h) d h=
$$$$
=\left|\int_{y}^{y+t}\left[e^{i \lambda h} g(h)-e^{-i \lambda h} g(-h)\right] \hat{u}(\lambda, y-h) d h\right| \leq 2 g_{\text {max }} \hat{u} \max |t|
$$

Because $g \in G$ then

$$
I(\lambda, p) \neq 0, \lambda \in R, \operatorname{Re} p \geq \rho .
$$

Therefore, we can multiply both sides of this equation by $\frac{1}{I(\lambda, p)}$, ie

where $g_{\max }=\sup |g(h)|$,

$$
\hat{u_{\max }}=\sup |\hat{u}(\lambda, y)|
$$

$$
\hat{\imath}(\lambda, p)=\frac{1}{I(\lambda, p)} \hat{f}(\lambda, p)
$$

We calculate the integral

$$
\int_{0}^{\infty} e^{-p \eta} f(\lambda, p) d \eta
$$

$2^{0} \hat{f}(\lambda, y)=0$ to all $y \leq 0$ and $\lambda \in R$. Indeed, since then, $u(x, t)=0 \quad \forall x \in R$ and $t \leq 0$, it is clear that $u(\lambda, t-h)=0$ for all $t \leq 0, \lambda \in R$ and $h \in[0, y]$;

$3^{0}$. The function $f(\lambda ;)$ has a zero growth rate. This fact follows from the finiteness $u(x, y)$ and mean value theorem.

Thus, $\hat{f}(\lambda, y)$ is a function of the original. Therefore according to Theorem 1(see[4]) to both sides of equation (7), as well as the equation (12)can be used the Laplace transform in $\mathrm{y}$.

Applicable to the equation(12)the Laplace Transform in the variable y. Using Fubini's theorem, we obtain:

$$
\begin{gathered}
\hat{f}(\lambda, p)=\int_{0}^{\infty} e^{-p y} * \\
*\left\{\int_{0}^{y}\left[e^{i \lambda h} g(h)-e^{-i \lambda h} g(-h)\right] \hat{u}(\lambda, y-h) d h\right\} d y= \\
=\int_{0}^{\infty} e^{-p(y-h)} \hat{u}(\lambda, y-h) d y \int_{0}^{\infty}\left[e^{i \lambda h} g(h)-e^{-i \lambda h} g(-h)\right] e^{-p h} d h= \\
\sim \hat{u}(\lambda, p) \int_{0}^{\infty}\left[e^{i \lambda h} g(h)-e^{-i \lambda h} g(-h)\right] e^{-p h} d h
\end{gathered}
$$

where $h=y-\eta$.

That means

$$
\hat{\hat{f}}(\lambda, p)=\hat{u}(\lambda, p) \int_{0}^{y}\left[e^{i \lambda h} g(h)-e^{-i \lambda h} g(-h)\right] e^{-p h} d h
$$

Further, using (3), we obtain the following equation:

Integrating by parts $n+2$ times and considering that $f(\cdot)$ finite in $\bar{\Omega}$ function, we arrive at the following relation:

$$
\int_{0}^{\infty} e^{-p \eta} f(\lambda, p) d \eta=\int_{0}^{\infty} \frac{1}{p^{n+2}} e^{-p \eta} \frac{\partial^{n+2} f(\xi, \eta)}{\partial \eta^{n+2}} d \eta .
$$

Equation (13) takes the form

$$
\hat{u}(\lambda, p)=\frac{1}{p^{n+2} I(\lambda, p)} * \int_{-\infty}^{\infty} \int_{0}^{\infty} e^{-p \eta} \frac{\partial^{n+2} f(\xi, \eta)}{\partial \eta^{n+2}} d \xi d \eta
$$

It follows from (6), the function $\frac{1}{p^{n+2} I(\lambda, p)}$ is analytic in the half plane $\operatorname{Re} p>0$ tends uniformly to zero at $|p| \rightarrow \infty$ in any half-plane $\operatorname{Re} p \geq a>0$ relative to $\arg p$ and integral

$$
\int_{p-i \aleph}^{a+i \infty} e^{p y} \frac{d p}{p^{n+2} I(\lambda, p)}
$$

is absolutely convergent. Consequently (see. [4]), the function $\left(p^{n+2} \cdot I(\lambda, p)\right)^{-1}$ is the Laplace transform of the function $I_{1}(\lambda, \cdot)$, more precisely

$$
I_{1}(\lambda, y-\eta)=\frac{1}{2 \pi i} \int_{a-i \infty}^{a-i \infty} e^{p(y-\eta)} \frac{d p}{p^{n+2} I(\lambda, p)} .
$$

Apply to both sides of equation (13) the inverse Laplace transform to the variable $p$ using the theorem circulation and convolution theorem, and also considering the properties of the Laplace transform. We have 


$$
\begin{aligned}
& \hat{u}(\lambda, y)=\int_{a-i \infty}^{a+i \infty} e^{p y} \int_{-\infty}^{\infty} \int_{0}^{\infty} \frac{1}{I(\lambda, p)} e^{-p \eta} \frac{1}{p^{n+2}} e^{-p \eta} * \\
& * \frac{\partial^{n+2} f(\xi, \eta)}{\partial \eta^{n+2}} e^{i \lambda \xi} d \xi d \eta d p= \\
& =\int_{0}^{\infty} \int_{-\infty}^{\infty} \int_{a-i \infty}^{a+i \infty} \frac{e^{p y}}{I(\lambda, p)} \frac{1}{p^{n+2}} e^{-p \eta} * \\
& * \frac{\partial^{n+2} f(\xi, \eta)}{\partial \eta^{n+2}} e^{i \lambda \xi} d p d \xi d \eta= \\
& =\int_{0}^{\infty} \int_{-\infty}^{\infty} \int_{a-i \infty}^{a+i \infty} \frac{e^{p(y-\eta)}}{p^{n+2} I(\lambda, p)} d p \frac{\partial^{n+2} f(\xi, \eta)}{\partial \eta^{n+2}} * e^{i \lambda \xi} d \xi d \eta= \\
& \int_{0}^{\infty} \int_{-\infty}^{\infty} I_{1}(\lambda, y-\eta) \frac{\partial^{n+2} f(\xi, \eta)}{\partial \eta^{n+2}} e^{i \lambda \xi} d \xi d \eta .
\end{aligned}
$$

So

$$
\hat{u}(\lambda, y)=\int_{0}^{\infty} \int_{-\infty}^{\infty} I_{1}(\lambda, y-\eta) \frac{\partial^{n+2} f(\xi, \eta)}{\partial \eta^{n+2}} e^{i \lambda \xi} d \xi d \eta .
$$

Divide and multiply the right-hand side of equation (15) by $\left(1+\lambda^{m+2}\right)$ :

$$
\begin{gathered}
\hat{u}(\lambda, y)=\int_{0}^{\infty} \int_{-\infty}^{\infty}\left(1+\lambda^{m+2}\right) I_{1}(\lambda, y-\eta) * \\
\frac{\partial^{n+2} f(\xi, \eta)}{\left(1+\lambda^{m+2}\right) \partial \eta^{n+2}} e^{i \lambda \xi} d \xi d \eta .
\end{gathered}
$$

In the integral

$$
\int_{-\infty}^{\infty} \frac{\partial^{n+2} f(\xi, \eta)}{\partial \eta^{n+2}} e^{i \lambda \xi} d \xi
$$

Applying integration by parts $m+2$ times, we obtain

$$
\begin{gathered}
\int_{-\infty}^{\infty} \frac{\partial^{n+2} f(\xi, \eta)}{\partial \eta^{n+2}} e^{i \lambda \xi} d \xi= \\
=\frac{1}{(i \lambda)^{m+2}} \int_{-\infty}^{\infty} \frac{\partial^{n+m+4} f(\xi, \eta)}{\partial \eta^{n+2} \partial \xi^{m+2}} e^{i \lambda \xi} d \xi .
\end{gathered}
$$

From here(16) takes the form

$$
\begin{gathered}
\hat{u}(\lambda, y)=\int_{0}^{\infty} \frac{e^{-\lambda x} I_{1}(\lambda, y-\eta)}{\left(1+\lambda^{m+2}\right)}\left\{\int_{-\infty}^{+\infty} \frac{\partial^{n+2} f(\xi, \eta)}{\partial \eta^{n+2}} e^{i \lambda \xi} d \xi+\right. \\
\left.+\frac{1}{i^{m+2}} \int_{-\infty}^{\infty} \frac{\partial^{n+m+4} f(\xi, \eta)}{\partial \eta^{n+2} \partial \xi^{m+2}} e^{i \lambda \xi} d \xi\right\} d \eta .
\end{gathered}
$$

From (8) implies that the function $I_{1}(\lambda, y-\eta)\left(1+\lambda^{m+2}\right)^{-1}$ is the Fourier transform of the first variable function

$$
I_{2}(x-\xi, y-\eta)=\int_{-\infty}^{+\infty} \frac{e^{-i \lambda(x-\xi)} I_{1}(\lambda, y-\eta)}{\left(1+\lambda^{m}\right)} d \lambda
$$

Applying to the equation (18) the inverse Fourier transform to the variable $\lambda$ and using the convolution theorem and also property differentiation of the Fourier transform, we obtain:

$$
\begin{aligned}
& u(x, y)=\int_{-\infty}^{+\infty} \int_{0}^{\infty} \frac{e^{-i \lambda x} I_{1}(\lambda, y-\eta)}{\left(1+\lambda^{m+2}\right)}\left\{\int_{-\infty}^{+\infty} \frac{\partial^{n+2} f(\xi, \eta)}{\partial \eta^{n+2}} e^{i \lambda \xi} d \xi+\right. \\
& \left.+\frac{\lambda^{m+2}}{i^{m+2}} \int_{-\infty}^{\infty} \frac{\partial^{n+2} f(\xi, \eta)}{\partial \eta^{n+2}} e^{i \lambda \xi} d \xi\right\} d \eta d \lambda
\end{aligned}
$$

Using (9) and (17), equation (18) takes the form

$$
\begin{aligned}
& u(x, y)=\int_{-\infty}^{+\infty} \int_{0}^{\infty} \frac{e^{-i \lambda x} I_{1}(\lambda, y-\eta)}{\left(1+\lambda^{m+2}\right)}\left\{\int_{-\infty}^{+\infty} \frac{\partial^{n+2} f(\xi, \eta)}{\partial \eta^{n+2}} e^{i \lambda \xi} d \xi+\right. \\
& \left.+\frac{1}{i^{m+2}} \int_{-\infty}^{\infty} \frac{\partial^{n+m+4} f(\xi, \eta)}{\partial \eta^{n+2} \partial \xi^{m+2}} e^{i \lambda \xi} d \xi\right\} d \eta d \lambda= \\
& =\int_{0}^{+\infty} \int_{-\infty}^{\infty} \int_{-\infty}^{\infty} \frac{e^{-i \lambda(x-\xi)} I_{1}(\lambda, y-\eta)}{\left(1+\lambda^{m+2}\right)} * \\
& * d \lambda\left\{\frac{\partial^{n+2} f(\xi, \eta)}{\partial \eta^{n+2}}+\frac{1}{i^{m+2}}\right. \\
& \left.* \frac{\partial^{n+m+4} f(\xi, \eta)}{\partial \eta^{n+2} \partial \xi^{m+2}}\right\} d \xi d \eta= \\
& =\int_{0}^{\infty} \int_{-\infty}^{+\infty} I_{2}(x-\xi, y-\eta)\left\{\frac{\partial^{n+2}}{\partial \eta^{n+2}}+,\right. \\
& \left.+\frac{1}{i^{m+2}} \frac{\partial^{n+m+4}}{\partial \eta^{n+2} \partial \xi^{m+2}}\right\} f(\xi, \eta) d \xi d \eta
\end{aligned}
$$

Therefore,

$$
\begin{aligned}
& u(x, y)=\int_{0-\infty}^{\infty+\infty} \int_{-\infty} I_{2}(x-\xi, y-\eta)\left\{\frac{\partial^{n+2}}{\partial \eta^{n+2}}+\right. \\
& \left.+\frac{1}{i^{m+2}} \frac{\partial^{n+m+4}}{\partial \eta^{n+2} \partial \xi^{m+2}}\right\} f(\xi, \eta) d \xi d \eta .
\end{aligned}
$$

This means that

$$
\begin{aligned}
& u(x, y)=\int_{0}^{\infty+\infty} \int_{-\infty}^{\infty} I_{2}(x-\xi, y-\eta)\left\{\frac{\partial^{n+2}}{\partial \eta^{n+2}}+\right. \\
& \left.+\frac{1}{i^{m+2}} \frac{\partial^{n+m+4}}{\partial \eta^{n+2} \partial \xi^{m+2}}\right\} f(\xi, \eta) d \xi d \eta .
\end{aligned}
$$


Equation (19) has a local character in the variable $y$. In view the conditions $\operatorname{supp} u \subset \Omega$ it is clear, that the representation (19) for the solution of the equation (6) holds and by $l<\infty$. Then from (4), (12) and (19) implies uniqueness of the solution of the original problem $I$ in the class of functions $C_{0}^{2}(\Omega)$

Note that

$$
\begin{aligned}
& \|u\|_{L_{2}(\Omega)}=\int_{0}^{\infty} \int_{-\infty}^{+\infty}|u(x, y)|^{2} d x d y= \\
& =\int_{0}^{\infty} \int_{-\infty}^{+\infty} \mid \int_{0}^{\infty+\infty} \int_{-\infty}^{\infty} I_{2}(x-\xi, y-\eta)\left\{\frac{\partial^{n+2}}{\partial \eta^{n+2}}+\right. \\
& \left.+\frac{1}{i^{m+2}} \frac{\partial^{n+m+4}}{\partial \eta^{n+2} \partial \xi^{m+2}}\right\}\left.f(\xi, \eta) d \xi d \eta\right|^{2} d x d y \leq \\
& \leq\left.\int_{0}^{\infty} \int_{-\infty}^{+\infty}\left|\int_{0}^{\infty+\infty} \int_{-\infty}^{+\infty}\right| I_{2}(x-\xi, y-\eta)\right|^{2} d \xi d \eta \cdot \int_{0}^{\infty} \int_{-\infty}^{+\infty} \mid\left\{\frac{\partial^{n+2}}{\partial \eta^{n+2}}+\right. \\
& \left.\left.+\frac{1}{i^{m+2}} \frac{\partial^{n+m+4}}{\partial \eta^{n+2} \partial \xi^{m+2}}\right\}\left.f(\xi, \eta) d \xi d \eta\right|^{2}\right\} d x d y \leq \\
& \leq 2\left\|I_{2}\right\|_{L_{2}(\bar{\Omega})}\|f\|_{W_{2}^{n+2, m+2} \cdot M(D) .}
\end{aligned}
$$

From equation (12), using (4), it is easy to obtain the following inequality

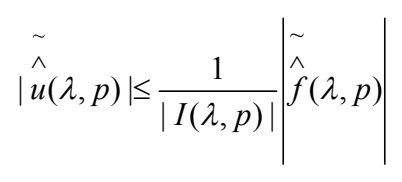

Which implies estimate

$$
\begin{gathered}
\int_{-\infty}^{+\infty} \int_{a-i \infty}^{a+i \infty}|\tilde{u}(\lambda, p)|^{2} d p d \lambda \leq \\
\leq \int_{-\infty}^{+\infty} \int_{a-i \infty}^{a+i \infty} \frac{1}{\left|I_{2}\right|^{2}}|\hat{f}(\lambda, p)|^{2} d p d \lambda
\end{gathered}
$$

Using the properties of differentiation Laplace and Fourier transforms, the triangle inequality for the norm, as well as taking into account (19) and (21, and the conditions imposed on the function $u$, we obtain the estimate

$$
\|u(x, y)\|_{L_{2}(\Omega)} \leq C_{0}\|f\|_{W_{2}^{n+2, m+2}(\Omega)},
$$

where $C_{0}$-is a constant Hence the uniqueness of the solution I.

\section{Theorem 1 is proved.}

\section{Uniqueness and Stability Problems with Indignation}

Now examine the problem of integral geometry with indignation.

Through $S(x, y)$ denote the part $R_{+}^{2}$, limited polyline $P(x, y)$ and axis $y=0$.

$\bar{\Omega}$ there is a stripe:

$$
\bar{\Omega}=\left\{(x, y): x \in R^{1}, y \in[0, l]\right\}
$$

Task II. Define a function $u(x, y)$ if for all $(x, y) \in \bar{\Omega}$ are known integrals from it for polyline $P(x, y)$ and squares $S(x, y)$ with a weight function $k(x, y ; \xi, \eta)$

$$
\begin{aligned}
& \int_{0}^{y} g(h) u(x-h, y+h) d h-\int_{0}^{y} g(-h) u(x+h, y-h) d h+ \\
& \quad+\int_{0}^{y} \int_{x-h}^{x+h} k(x, y ; \xi, \eta) u(\xi, \eta) d \xi d \eta=F(x, y),
\end{aligned}
$$

where $h=y-\eta$,

$$
g(h)=\left\{\begin{array}{llc}
a_{1} e^{-k h}, & \text { when } & h \geq 0, \\
-a_{1} e^{k h}, & \text { when } & h<0,
\end{array}\right.
$$

Function $k(x, y ; \xi, \eta)$ - a finite function has all of continuous partial derivatives up to second order inclusive and together with its derivatives becomes zero on polyline:

$$
\begin{gathered}
P(x, y)=\{(\xi, \eta): \quad(x-y)+(\eta-\xi)=0,0 \leq \eta \leq y, x-y \leq \xi \leq x\} \cup \\
\cup\{(\xi, \eta): \quad(x+y)-(\xi+\eta)=0,0 \leq \eta \leq y, x \leq \xi \leq x+y\}
\end{gathered}
$$

Function $F(x, y)$ is considered known throughout the half-plane.

Equation (22) corresponds to an integral geometry problem with indignation.

The first term in the left side (22)

$$
\begin{aligned}
& \int_{0}^{y} g(h) u(x-h, y+h) d h-\int_{0}^{y} g(-h) u(x+h, y-h) d h=f(x, y) \\
& \text { Where } h=y-\eta \text { represents set of integrals of the desired } \\
& \text { function from the family halves of the polyline with } \\
& \text { vertices at points }(x, y)
\end{aligned}
$$

The second term $f_{0}(x, y)=F(x, y)-f(x, y)$ - integral with weight $k(x, y ; \xi, \eta)$ in parts half-plane, limited polyline of the family $P(x, y)$.

Theorem 2. Let the function $F(x, y)$ is known in the strip $\Omega$.

The weight function $k(x, y ; \xi, \eta) \in C_{0}^{2}(\Omega \times \Omega)$ together 
with its derivatives up to second order inclusive becomes zero on the polyline $P(x, y)$

Then the decision of Problem II in the class of twice continuously differentiable and of finite functions the only in the strip $\Omega$ and executed the inequality

$$
\|u\|_{L_{2}(\Omega)} \leq C_{1}\|F\|_{W_{2}^{n+2, m+2}(\Omega)}
$$

Where $C_{1}$-is some constant.

Of the conditions, which imposed on the functions $u(x, y)$ and $k(x, y ; \xi, \eta)$ it follows that the function $f(x, y)$ and $F(x, y)$ will have all continuous derivatives up to second order inclusive.

\section{The proof of Theorem 2.}

Consider the function $f_{0}(x, y)=F(x, y)-f(x, y)$, that is, the second term of the left side of equation (22):

$$
\int_{0}^{y} \int_{x-h}^{x+h} k(x, y ; \xi, \eta) u(\xi, \eta) d \xi d \eta=f_{0}(x, y),
$$

where $h=y-\eta$.

The left side of equation (23) is integral with the weight $k(x, y ; \xi, \eta)$ in parts half-plane, limited polyline from the family $P(x, y)$. From the conditions, imposed on the function $u(x, y)$ and $k(x, y ; \xi, \eta)$, follows that the function $f_{0}(x, y)$ will be continuous together with its partial derivatives up to second order inclusive.

Considering that the function $k(x, y ; \xi, \eta)$ together with its partial derivatives up to second order becomes zero on the polyline $P(x, y)$ we have:

$$
\begin{aligned}
& \frac{\partial f_{0}(x, y)}{\partial x}=\int_{0}^{y} \int_{x-h}^{x+h} k_{x}(x, y ; \xi, \eta) u(\xi, \eta) d \xi d \eta, \\
& \frac{\partial^{2} f_{0}(x, y)}{\partial x^{2}}=\int_{0}^{y} \int_{x-h}^{x+h} k_{x x}(x, y ; \xi, \eta) u(\xi, \eta) d \xi d \eta .
\end{aligned}
$$

For $y<y_{0}$ where $y_{0}$ is small enough, out the formulas (24) - (25), considering the restrictions, imposed on the weight function $k(x, y ; \xi, \eta)$, and using the expressions for the corresponding derivatives of the function $f_{0}(x, y)$, we obtain the estimate

$$
\left\|f_{0}(x, y)\right\|_{W_{2}^{n+2, m+2}(\Omega)} \leq \varepsilon\|u(x, y)\|_{L_{2}(\Omega)},
$$

where $\varepsilon \rightarrow 0$ by $y_{0} \rightarrow 0$

Of the estimates, obtained in the theorem 1, and the triangle inequality for the norms it follows, that

$$
\begin{gathered}
\|u(x, y)\|_{L_{2}(\Omega)} \leq C_{0}\left(\|F\|_{W_{2}^{n+2, m+2}(\Omega)}+\right. \\
\left.+\left\|f_{0}\right\|_{W_{2}^{n+2, m+2}(\Omega)}\right)
\end{gathered}
$$

Using (26) and (27), it is easy to obtain the inequality

$$
\|u(x, y)\|_{L_{2}(\Omega)} \leq C_{1}\|F(x, y)\|_{W_{2}^{n+2, m+2}(\Omega)},
$$

where

$$
C_{1}=\frac{C_{0}}{1-C_{0} \varepsilon} .
$$

Out of (26), (28) and (29) implies the uniqueness of solutions of the equation (22) for sufficiently small $y$. And because equation (22) is the equation of Volterrain the sense of the definition, given in [3], that explicitness and stability estimate (28) will take place not only small $y$, but also in the whole stripe $\Omega$.

\section{Theorem 2 is proved.}

\section{REFERENCES}

[1] M.M. Lavrentiev, V.G. Romanov, S.P. Shishatskii. Ill-posed problems of mathematical physics and analysis. M.:, Science, 1980.

[2] M.M. Lavrentiev. Integral geometry and inverse problems// ill-posed problems of mathematical physics and analysis. Novosibirsk.: Science,1984, pp. 81-86.

[3] M.M. Lavrentiev, Saveliev L.Y. Operator The or y and ill-posed problems. - Sobolev Institute of Mathematics, Russian Academy of Sciences, Novosibirsk, Russia.1999. $-702 \mathrm{p}$.

[4] Lavrentiev M.M, B.V. Shabat. Methods of the theory of functions of a complex variable. - M .: Science., 1986

[5] V.G. Romanov. Some inverse problems for equations of hyperbolic type. Novosibirsk. Science. 1974

[6] Akram H. Begmatov. Two classes weakly ill-posed problems of integral geometry on a plane.// Sib.mat.journal.1995. Vol.36. No 2, pp. 243-247.

[7] Akram H. Begmatov. On a class of Weakly ill-posed problems of integral geometry in three-dimensional space. // J. Inverse and Ill-Posed problems. 1995. Vol. 3, pp. 231-235.

[8] Akram H. Begmatov. Integral geometry problem for a family of cones inn-dimensional space.// Sib.mat.journal.1997. Vol.38. No 4, pp. 723-737.

[9] Akbar H. Begmatov. O uniqueness of the solution Volterra type integral geometry in the plane.// DAN. 2009. Vol. 427. No 2, pp. 439-441.

[10] Akbar H.Begmatov, N.N. Petrova. The problem of integral geometry with indignation on elliptic curves in the strip//Doklady Mathematics. - 2011. - Vol. 83. No 1, pp. $22-25$ 
[11] Akram H. Begmatov, Z.H. Ochilov. Integral Geometry Problem with a Discontinuous Weight Function. Doklady Mathematics, 2009, Vol. 80, No. 3, pp. 823-825.

[12] Akbar H. Begmatov and Akram H. Begmatov. Problems of integral geometry on curves and surfaces in Euclidean space // Ill-Posed and Non-Classical Problems of Mathematical
Physics and Analysis, M.M. Lavrente'v et al., Eds., Proceedings of International Conference, VSP, Utrecht-Boston, 2003, pp.1-18.

[13] Akbar H. Begmatov. The problems of integral geometry with indignation in three dimensional space//Sib.mat.journal, 2000. Vol.41. No1, pp. 3-14. 